\title{
MATLAB-aided teaching and learning in optics and photonics using the methods of computational photonics
}

Zhili Lin, Xiaoyan Li, Daqing Zhu, Jixiong Pu

Zhili Lin, Xiaoyan Li, Daqing Zhu, Jixiong Pu, "MATLAB-aided teaching and learning in optics and photonics using the methods of computational photonics," Proc. SPIE 10452, 14th Conference on Education and Training in Optics and Photonics: ETOP 2017, 104521W (16 August 2017); doi: $10.1117 / 12.2270722$

SPIE Event: 14th Conference on Education and Training in Optics and Photonics, ETOP 2017, 2017, Hangzhou, China 


\title{
MATLAB-aided teaching and learning in optics and photonics using the methods of computational photonics
}

\author{
Zhili Lin*, Xiaoyan Li, Daqing Zhu and Jixiong Pu \\ Fujian Key Laboratory of Light Propagation and Transformation, College of \\ Information Science and Engineering, \\ Huaqiao University, Xiamen 361021, China \\ *E-mail: zllin2008@gmail.com
}

\begin{abstract}
Due to the nature of light fields of laser waves and pulses as vector quantities with complex spatial distribution and temporal dependence, the optics and photonics courses have always been difficult to teach and learn without the support of graphical visualization, numerical simulations and hands-on experiments. One of the state-of-the-art method of computational photonics, the finite-difference time-domain(FDTD) method, is applied with MATLAB simulations to model typical teaching cases in optics and photonics courses. The obtained results with graphical visualization in the form of animated pictures allow students to more deeply understand the dynamic process of light interaction with classical optical structures. The discussed teaching methodology is aimed to enhance the teaching effectiveness of optics and photonics courses and arousing the students' learning interest.
\end{abstract}

Key Words: Education in optics and photonics, MATLAB-aided teaching, computational photonics, finite-difference time-domain (FDTD) method

\section{INTRODUCTION}

The courses about optics and photonics are a kind of professional foundation compulsory courses for information science and engineering undergraduates. It is an important part of the knowledge structure system of undergraduates majored in information science and engineering. However, due to the abstract concept of electromagnetic and optical theories in such courses, the spatial and temporal distributions are complex, and students need strong abstract thinking ability and spatial imagination ability. If we follow the traditional classroom static teaching methods, students cannot form a dynamic physical picture and this not only makes the classroom teaching effect poor, time-consuming and laborious, but also easily cause

\footnotetext{
14th Conference on Education and Training in Optics and Photonics: ETOP 2017, edited by Xu Liu,

Xi-Cheng Zhang, Proc. of SPIE Vol. 10452, 104521W · C 2017 ICO, IEEE, OSA, SPIE

CCC code: $0277-786 X / 17 / \$ 18 \cdot$ doi: $10.1117 / 12.2270722$
} 
students to lose their interest in the process of learning. At the same time, the current optics and photonics courses place two much emphasis on the theory teaching and don't have corresponding actual experiments due to the lack of expensive equipment facility or place, resulting in students unable to master and accurately understand the optical waves and fields of the actual spatial distribution and dynamic space process. In order to solve the existing teaching problems in these courses and improve the teaching quality and teaching effect, we should try to explore the introduction of the methods of computational photonics as the new teaching methods, and actively carry out visual teaching research in such courses. At present, computer-aided teaching tools, represented by professional math software such as MATLAB, Mathematica and Maple, have played an important role in many science and engineering teaching. The use of professional mathematical software can be combined with theoretical teaching and visual teaching, but also can design a large number of simulation teaching experiments, so as to deepen students' understanding of optical theory and space-time imagination of electromagnetic fields. In this paper, we will analyze the classification and characteristics of the simulation function of Finite-difference Time-Domain (FDTD) method, a popular method of computational photonics, and explore its programming tactics in order to improve the visualization and attraction of teaching and learning in the relevant courses. Two examples with FDTD simulations are presented as two typical teaching cases in optics courses with MATALB graphical visualization in the form of animated pictures that allow students to more deeply understand the dynamic process of light propagation in classical optical structures.

\section{FDTD METHOD}

In the past several decades, the finite-difference time-domain (FDTD) method has become one of the most powerful numerical techniques in solving the Maxwell's curl equations [1] and has been widely applied to solve the complex optical and photonic problems [2]. FDTD modeling has many strength. FDTD is a versatile modeling technique used to solve Maxwell's equations. It is intuitive, so users can easily understand how to use it and know what to expect from a given model. The FDTD technique allows the user to specify the material at all points within the computational domain. Thus it can model any type of complicated structures. Nevertheless, a wide variety of dispersive dielectric and magnetic materials can be naturally and easily modeled. Since FDTD method calculates the electric and magnetic fields everywhere in the computational domain as they evolve in time, it lends itself to providing animated displays of the electromagnetic field movement through the model. Thus the obtained simulation results can be shown with graphical visualization by MATLAB in the form of animated pictures allow students to more deeply understand the dynamic process of light propagation in classical optics and

photonics structures. Such as for modelling a dispersive medium described by the 
Drude model, and the corresponding constitutive relation is rewritten as

$$
\boldsymbol{D}(\omega)=\varepsilon_{0}\left(1+\frac{\omega_{\mathrm{pe}}^{2}}{v_{\mathrm{e}}(\mathrm{j} \omega)+(\mathrm{j} \omega)^{2}}\right) \boldsymbol{E}(\omega)
$$

By applying the Maclaurin series expansion (MSE) approach proposed in [3] to realize the dispersive dielectric constant in the FDTD method, we have

$$
\boldsymbol{E}^{n+1}=\frac{b_{0} \boldsymbol{D}^{n+1}+b_{1} \boldsymbol{D}^{n}+b_{2} \boldsymbol{D}^{n-1}}{\varepsilon_{0}\left(a_{0} \bar{\omega}_{\mathrm{p}}^{2}+b_{0}\right)}-\frac{a_{1} \bar{\omega}_{\mathrm{p}}^{2}+b_{1}}{a_{0} \bar{\omega}_{\mathrm{p}}^{2}+b_{0}} \boldsymbol{E}^{n}-\frac{a_{2} \bar{\omega}_{\mathrm{p}}^{2}+b_{2}}{a_{0} \bar{\omega}_{\mathrm{p}}^{2}+b_{0}} \boldsymbol{E}^{n-1},
$$

where $\bar{\omega}_{\mathrm{p}}^{2}=\omega_{\mathrm{p} 0}^{2} \Delta t^{2}$ and the normalized coefficients

$$
\begin{gathered}
a_{0}=6+3 \bar{\nu}_{\mathrm{c}}-\bar{\nu}_{\mathrm{c}}^{2}, a_{1}=60-4 \bar{\nu}_{\mathrm{c}}^{2}, a_{2}=6-3 \bar{\nu}_{\mathrm{c}}-\bar{\nu}_{\mathrm{c}}^{2}, \\
b_{0}=72+36 \bar{\nu}_{\mathrm{c}}-3 \bar{\nu}_{\mathrm{c}}^{3}, b_{1}=-144, b_{2}=72-36 \bar{\nu}_{\mathrm{c}}+3 \bar{\nu}_{\mathrm{c}}^{3}
\end{gathered}
$$

with $\bar{v}_{\mathrm{c}}=v_{\mathrm{c}} \Delta t$. In fact, the accuracy of MSE approach is higher than the other approaches in view of the FDTD implementation of Drude model [4].

On the other hand, the optical wave sources are critically important for solving any optics and photonics problems. Since the total-field/scattered-field (TF/SF) technique can be applied based on the well-known equivalence principle [5], the FDTD method also offers a great degree of convenience and flexibility in the excitation of various types of optics wave sources [6]. Assume, without loss of generality, that the laser beam has the electromagnetic fields, $\boldsymbol{E}_{\mathrm{L}}(x, y, z, t)$ and $\boldsymbol{H}_{\mathrm{L}}(x, y, z, t)$. By utilizing the TF/SF source condition in the FDTD method, we can artificially generate arbitrary types of laser beams with the designed pattern and parameters. The TF/SF interface is perpendicular to the $z$ axis and locates at $z=\left(k_{\mathrm{s}}-1 / 4\right) \Delta z$, so that the tangential components of total electric field on the grid plane $z=k_{\mathrm{s}} \Delta z$ of YEE cells at the instant $t=(n+1) \Delta t$ can be updated by[7]

$$
D_{\mathrm{t}, x}^{(n+1)}\left(i+\frac{1}{2}, j, k_{\mathrm{s}}\right)=D_{\mathrm{t}, x}^{(n+1)}\left(i+\frac{1}{2}, j, k_{\mathrm{s}}\right)_{\mathrm{FDTD}}+\frac{\Delta t}{\Delta z} H_{\mathrm{L}, y}^{(n+1 / 2)}\left(i+\frac{1}{2}, j, k_{\mathrm{s}}-\frac{1}{2}\right)
$$

with the $y$ component of the magnetic field of the incident laser beam 


$$
H_{\mathrm{L}, y}^{(n+1 / 2)}\left(i+\frac{1}{2}, j, k_{\mathrm{s}}-\frac{1}{2}\right)=H_{\mathrm{L}, y}\left[\left(i+\frac{1}{2}\right) \Delta x, j \Delta y,\left(k_{\mathrm{s}}-\frac{1}{2}\right) \Delta z,\left(n+\frac{1}{2}\right) \Delta t\right],
$$

and

$$
D_{\mathrm{t}, y}^{(n+1)}\left(i, j+\frac{1}{2}, k_{\mathrm{s}}\right)=D_{\mathrm{t}, y}^{(n+1)}\left(i, j+\frac{1}{2}, k_{\mathrm{s}}\right)_{\mathrm{FDTD}}-\frac{\Delta t}{\Delta z} H_{\mathrm{L}, x}^{(n+1 / 2)}\left(i, j+\frac{1}{2}, k_{\mathrm{s}}-\frac{1}{2}\right)
$$

with the $x$ component of the magnetic field of the incident laser beam

$$
H_{\mathrm{L}, x}^{(n+1 / 2)}\left(i, j+\frac{1}{2}, k_{\mathrm{s}}-\frac{1}{2}\right)=H_{\mathrm{L}, x}\left[i \Delta x,\left(j+\frac{1}{2}\right) \Delta y,\left(k_{\mathrm{s}}-\frac{1}{2}\right) \Delta z,\left(n+\frac{1}{2}\right) \Delta t\right] .
$$

On the other hand, the tangential components for the scattering magnetic field on the grid plane $z=\left(k_{\mathrm{s}}-1 / 2\right) \Delta z$ at the instant $t=(n+1 / 2) \Delta t$ can be updated by

$$
B_{\mathrm{s}, x}^{(n+1 / 2)}\left(i, j+\frac{1}{2}, k_{\mathrm{s}}-\frac{1}{2}\right)=B_{\mathrm{s}, x}^{(n+1 / 2)}\left(i, j+\frac{1}{2}, k_{\mathrm{s}}-\frac{1}{2}\right)_{\mathrm{FDTD}}-\frac{\Delta t}{\Delta z} E_{\mathrm{L}, y}^{(n)}\left(i, j+\frac{1}{2}, k_{\mathrm{s}}\right)
$$

with the $y$ component of the electric field of the incident laser beam

$$
E_{\mathrm{L}, y}^{(n)}\left(i, j+\frac{1}{2}, k_{\mathrm{s}}\right)=E_{\mathrm{L}, y}\left[i \Delta x,\left(j+\frac{1}{2}\right) \Delta y, k_{\mathrm{s}} \Delta z, n \Delta t\right]
$$

and

$$
B_{\mathrm{s}, y}^{(n+1 / 2)}\left(i+\frac{1}{2}, j, k_{\mathrm{s}}-\frac{1}{2}\right)=B_{\mathrm{s}, y}^{(n+1 / 2)}\left(i+\frac{1}{2}, j, k_{\mathrm{s}}-\frac{1}{2}\right)_{\mathrm{FDTD}}+\frac{\Delta t}{\Delta z} E_{\mathrm{L}, x}^{(n)}\left(i+\frac{1}{2}, j, k_{\mathrm{s}}\right)
$$

with the $x$ component of the electric field of the incident laser beam

$$
E_{\mathrm{L}, y}^{(n)}\left(i, j+\frac{1}{2}, k_{\mathrm{s}}\right)=E_{\mathrm{L}, y}\left[i \Delta x,\left(j+\frac{1}{2}\right) \Delta y, k_{\mathrm{s}} \Delta z, n \Delta t\right] .
$$

Under this TF/SF source condition, we can numerically generate arbitrary types of optical waves with the designed pattern and parameters.

\section{TEACHING CASES}

\subsection{Total reflection at a diamond -air interface}

The first teaching case is to study the phenomenon of total reflection at a diamond-air interface. The diamond is with refractive index 2.42 for a Gaussian beam with wavelength $\lambda_{0}=589 \mathrm{~nm}$. The Gaussian beam is with a waist width $w_{0}=2 \lambda_{0}$ and is incident on the interface with an incident angle $45^{\circ}$, in which case the total reflection 
occurs. The FDTD simulation results about the transient and amplitude of the electric field of the Gaussian beam that are shown in Fig.1. It is apparent that the Gaussian beam does experience total reflection for an incident angle larger than critical angle.
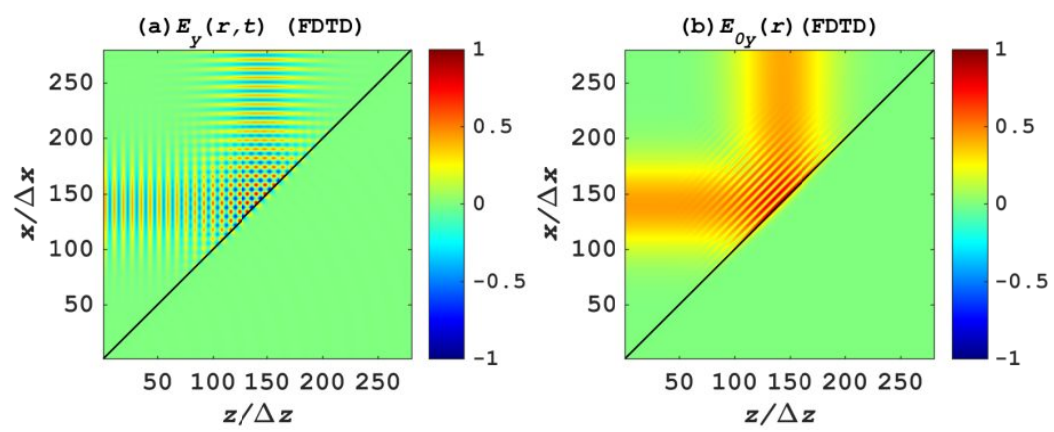

Fig. 1. The FDTD simulation results about the (a) transient and (b) amplitude of the electric field of a Gaussian beam incident on the diamond-air interface with an incident angle of $45^{\circ}$.

\subsection{Optical wave scattering from a dielectric cylinder}

The second teaching case is to show a y-polarized uniform plane wave with amplitude $E_{0 y}=1 \mathrm{~V} / \mathrm{m}$ and wavelength $\lambda_{0}=589.29 \mathrm{~nm}$ propagating in air and scattered by a circular fused-quartz cylinder with radius $R=2.5 \lambda_{0}[8]$. The total sizes of the computation region is $16 \lambda_{0} \times 16 \lambda_{0}$ and also surrounded by 20 -layer PMLs. The spatial and temporal discretization parameters are $N_{\lambda}=20, n_{\mathrm{CFL}}=1 / 2$ and $N_{T}=40$. The simulation results are illustrated in Fig. 2. However, this problem has the analytical solution for the steady state calculated by using the formulas in Section 6.4.3 of Ref. [9] Fig. 3 show the FDTD simulation results when the steady sate is

Fig. 2. Snapshots of the electric field of a plane light wave scattered by a circular dielectric cylinder at different time instants extracted from the FDTD simulations.
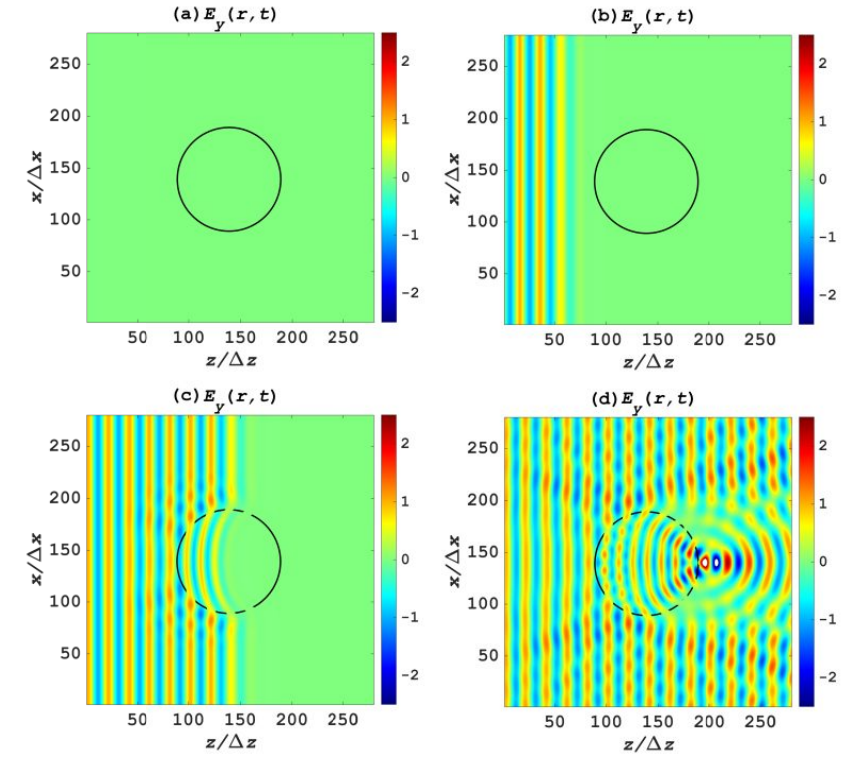

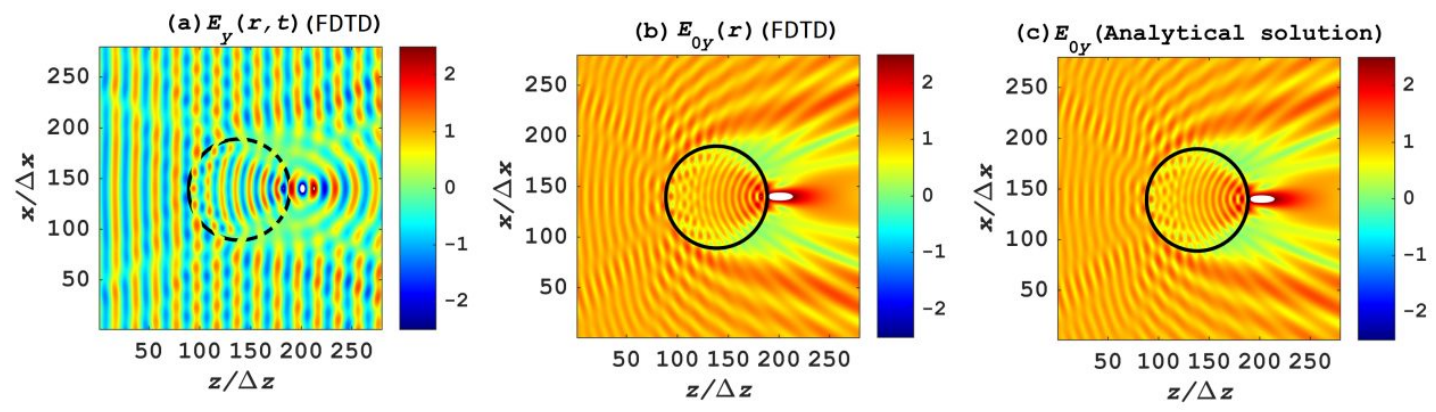

Fig. 3. The FDTD simulation results about (a) the instantaneous electric field; (b) the amplitude distribution extracted by the FDTD algorithm, (c) the analytical solution of amplitude distribution calculated by the formula in Section 6.4.3 of Ref. [9].

reached. Fig. 3 (b) and (c) show the simulation and analytical solution to the amplitude of the scattered waves, where the series summation is evaluated from $n=-200$ to $n=200$. It can be seen that the FDTD method is highly accurate for this type of simulation and suitable for modeling the phenomena of light scattering.

\section{CONCLUSION}

The optics and photonics courses have always been difficult to teach and learn without the support of graphical visualization, numerical simulations and hands-on experiments. The finite-difference time-domain(FDTD) method combined with MATLAB is introduced to model typical teaching cases in optics and photonics courses. The FDTD method has many strengths that can be utilized to improve the visualization and attraction of teaching and learning in the optical and photonics courses. The obtained results with graphical visualization in the form of animated pictures allow students to more deeply understand the dynamic interaction of light propagation with classical optical structures. The discussed teaching methodology has a beneficial effect on enhancing the teaching effectiveness of optics and photonics courses and arousing the students' learning interest.

\section{ACKNOWLEDGMENTS}

This work is supported by the Undergraduate Education and Teaching Reform Research Project of Fujian Province (No: JZ160081) and the Undergraduate Education and Teaching Reform Key Project of Huaqiao University (No: 15JGZD08). 


\section{REFERENCES}

[1] Taflove A. and Hagness, S. C., [Computational Electrodynamics: The Finite-Difference Time-Domain Method], Artech House, Norwood, MA (2005).

[2] Taflove, A., Oskooi, A., and Johnson, S. G., [Advances in FDTD Computational Electrodynamics: Photonics and Nanotechnology], Artech House, Norwood, MA (2013).

[3] Lin, Z., Zhang, C., Ou, P., Jia, Y., and Feng, L., "A generally optimized FDTD model for simulating arbitrary dispersion based on the Maclaurin series expansion," J. Lightwave Technol. 28(19), 2843-2850 (2010).

[4] Lin, Z., Ou, P., Jia, Y., and Zhang, C., "A highly accurate FDTD model for simulating Lorentz dielectric dispersion," IEEE Photonics Technology Letters 21(22), 1692-1694 (2009).

[5] Merewether, D. E., Fisher, R., and Smith, F. W., "On implementing a numeric Huygen's source scheme in a finite difference program to illuminate scattering bodies," IEEE Trans. Nucl. Sci. 27(6), 1829-1833 (1980).

[6] Tan, T. and Potter, M., "FDTD discrete planewave (FDTD-DPW) formulation for a perfectly matched source in TFSF simulations," IEEE Trans. Antenna Propagat. 58(8), 2641-2648 (2010).

[7] Lin, Z., "Interaction of high power laser beams with plasma in ICF hohlraum using the FDTD method," Proc. of SPIE, 10016, 100160W-11 (2016).

[8] Lin, Z., Zhu, D., and Pu, J., "Research on MATLAB animation techniques in visual teaching of electromagnetic theory courses," China Modern Educational Equipment, 259, 30-32 (2017).

[9] Jin, J.-M., [Theory and computation of electromagnetic fields], John Wiley \& Sons, Hoboken, NJ (2015). 